\title{
MAPK signaling in inflammation-associated cancer development
}

\author{
Pengyu Huang ${ }^{1}$, Jiahuai $\operatorname{Han}^{2}{ }^{\bowtie}$, Lijian Hui $^{1 凶}$ \\ ${ }^{1}$ Laboratory of Molecular Cell Biology, Institute of Biochemistry and Cell Biology, Shanghai Institutes for Biological Sciences, \\ Chinese Academy of Sciences, Shanghai 200031, China \\ 2 Key Laboratory of the Ministry of Education for Cell Biology and Tumor Cell Engineering, School of Life Sciences, Xiamen \\ University, Xiamen 361005, China \\ \ Correspondence: jhan@xmu.edu.cn (J.Han), ljhui@sibs.ac.cn (L.Hui)
}

Received November 26, 2009 Accepted December 9, 2009

\begin{abstract}
Mitogen-activated protein (MAP) kinases comprise a family of protein-serine/threonine kinases, which are highly conserved in protein structures from unicellular eukaryotic organisms to multicellular organisms, including mammals. These kinases, including ERKs, JNKs and p38s, are regulated by a phosphorelay cascade, with a prototype of three protein kinases that sequentially phosphorylate one another. MAPKs transduce extracellular signals into a variety of cellular processes, such as cell proliferation, survival, death, and differentiation. Consistent with their essential cellular functions, MAPKs have been shown to play critical roles in embryonic development, adult tissue homeostasis and various pathologies. In this review, we discuss recent findings that reveal the profound impact of these pathways on chronic inflammation and, particularly, inflammation-associated cancer development.
\end{abstract}

\section{INTRODUCTION}

The mitogen activated protein kinases (MAPKs) are a family of signal transduction proteins that convert extracellular signals, such as stresses and growth factors, to the activation of intracellular pathways. The signaling transduction pathways of MAPKs have been highly conserved from yeast to multicellular organisms. In mammals, at least four subfamilies of MAPKs have been described, including the extracellular signal-regulated kinases (ERKs), the c-Jun NH2-terminal kinases (JNKs), the p38 isoforms (p38s), and ERK5 (Pearson et al., 2001; Boutros et al., 2008; Wagner and Nebreda, 2009) (Figure 1). MAPKs are mainly modulated by upstream MAPK kinases (MKKs) and MAPK phosphatases that modify the phosphorylation of the MAPK threonine and tyrosine (T-X-Y) motif. Activated MAPKs catalyze the phosphorylation on specific serine and threonine residues of target substrates and trigger a wide range of cellular responses, such as proliferation, differentiation, and apoptosis. In consistence with their important roles in cellular processes, MAPKs have been found to play key physiological roles, such as regulating embryonic development and maintaining tissue homeostasis. On the other hand, extracellular stresses can induce MAPK activation in pathological conditions. For example, there are accumulative data showing that MAPK activation is critical in regulating inflammation-associated cancer development (Wagner and Nebreda, 2009). Moreover, MAPKs have been considered promising targets for therapies, and great efforts are therefore being made to explore new functions and mechanisms regulated by MAPKs in disease conditions. In this review, we aim to summarize current knowledge about the signaling transduction of ERK1/2, JNKs, and p38s MAPK members and their roles in inflammation and cancer development.

\section{MAPK SIGNALING}

\section{ERK1/2}

Extracellular signal-regulated kinases $1 / 2$ (ERK1/2) are homologous isoforms which are expressed in nearly all tissues (Chambard et al., 2007; Mebratu and Tesfaigzi, 2009). ERK1 and ERK2, with $83 \%$ shared protein sequences, are regulated by similar molecular signals and have overlapping roles in many aspects. However, distinguished functions have been characterized, particularly by work on ERK1 and ERK2 knockout mice. ERK1-null mice are viable and fertile; however, thymocyte maturation is impaired in these mice 


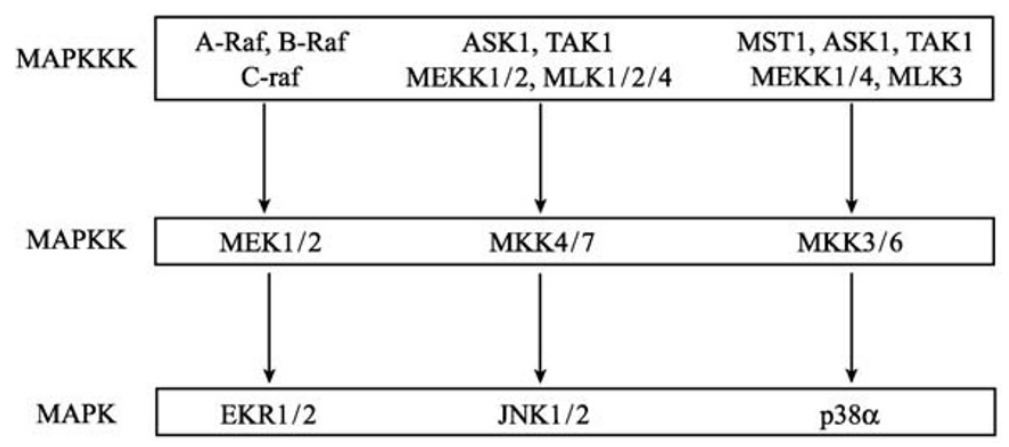

Figure 1. An over-simplified diagram of the classical MAP kinase pathways in mammals. The classical MAP kinase pathways consist of a module of three kinases that subsequently phosphorylate one another: the MAP kinase kinase kinase (MAPKKK) is the upstream activator of MAP kinase kinase (MAPKK), which in turn phosphorylates MAP kinase (MAPK). Interactions between the different MAPK pathways are not depicted in this diagram, but are discussed in the text.

(Pages et al., 1999). Other studies also show that ERK1-null mice have increased locomotor activity (Mazzucchelli et al., 2002) and impaired adipocyte differentiation (Bost et al., 2005). In contrast, ERK2-null mice show completely different phenotypes; these mice die at embryonic day 6.5-8.5 due to impaired mesoderm differentiation and placental development (Hatano et al., 2003; Saba-El-Leil et al., 2003; Yao et al., 2003).

It has been widely accepted that many extracellular activators, such as hormones and growth factors, activate ERK1/2 largely through the Ras-Raf-MEK module (Chambard et al., 2007). Upon activation by extracellular signals, receptor tyrosine kinases located in the plasma membrane can induce the phosphorylation of Ras, which recruits and phosphorylates Raf (Chambard et al., 2007). Consequently, Raf phosphorylates downstream MEK1/2, which further activates ERK1/2 by phosphorylation on the Thr-Glu-Tyr (TEY) motif (Chambard et al., 2007).

Activation of the ERK pathway has been found to function in proliferation, cell death, and cytoskeletal remodeling, by translocating to the nucleus and activating transcript factors (Chambard et al., 2007; Dumesic et al., 2009; Pullikuth and Catling, 2007). Both transient and sustained activations of ERK1/2 phosphorylate downstream Elk and induce c-Fos expression during cell cycle procession (Chambard et al., 2007; Dumesic et al., 2009). In addition, ERK1/2 are implicated in the stabilization of c-Myc, which together with Max upregulate cyclin D1 during the G1-S transition (Chambard et al., 2007; Dumesic et al., 2009). Moreover, ERK $1 / 2$ can activate CDK proteins, which in turn phosphorylate the retinoblastoma protein and induce E2F-mediated transcription of cell cycle-related genes (Chambard et al., 2007; Dumesic et al., 2009). The ERK signaling pathways also play important roles in regulating cell shape and motility. Emerging data indicate ERK signaling in controlling the dynamics of F-actin and focal adhesion formation during cell morphogenesis and migration. ERK scaffolding proteins also appear important in transmitting signals to adhesion turnover sites. For more details, please refer to the review by Pullikuth and Catling (2007).

\section{JNKs}

JNKs consist of at least ten isoforms derived from alternatively spliced mRNAs of three genes: JNK1, JNK2, and JNK3 (Wagner and Nebreda, 2009; Weston and Davis, 2007). JNK1 and JNK2 are ubiquitously expressed, whereas JNK3 is mainly found in the brain. The genes that encode JNKs have been disrupted by homologous recombination in mice. Mice lacking either one of the JNKs are viable; however, they show impaired apoptosis and immune responses. When JNK1 and JNK2 are both deleted, these mice display early embryonic lethality associated with exencephaly (Kuan et al., 1999; Sabapathy et al., 1999).

Dual phosphorylation on the Thr-Pro-Tyr (TPY) motif of JNKs, which was first found in UV-stimulated cells, is responsible for the activation of JNKs (Weston and Davis, 2007; Wagner and Nebreda, 2009). Upstream kinases MKK4 and MKK7 phosphorylate JNK on tyrosine and threonine (Dong et al., 2000; Lawler et al., 1998; Tournier et al., 2001). Both MKK4 and MKK7 are required for full activation of JNKs, since overexpression of MKK4 could not substitute reduced proliferation of MKK7-null cells and vice versa (Wada et al., 2004). MKK4 and MKK7 are in turn phosphorylated and activated by a large number of upstream kinases known as MAP3Ks, including MEKK1, MEKK2, MLK1, MLK2, and MLK4 (Weston and Davis, 2007; Wagner and Nebreda, 2009).

JNKs are involved in cell proliferation and apoptosis through activating various targets. A well-known group of substrates of JNK is the AP-1 transcript factors that include Fos and Jun family members (Eferl and Wagner, 2003). However, different JNKs might be required in regulating the same AP-1 gene under different conditions. For example, cJun is targeted by JNK2 for its ubiquitination and degradation in quiescent cells, whereas JNK1-mediated phosphorylation 
of c-Jun on Ser73 is essential for protecting c-Jun from ubiquitination under stress (Fuchs et al., 1996; Sabapathy et al., 2004). Recent data also show that elevated JNK activities promote proliferation of mouse fibroblasts (Sabapathy et al., 2004; Jaeschke et al., 2006). However, regarding the function of specific JNK isoforms in proliferation, controversial observations were reported, probably due to the use of different experimental systems (Sabapathy et al., 2004; Jaeschke et al., 2006). The molecular mechanisms underlying the pro-apoptotic functions of JNKs are dependent on cellular context and stimuli. Studies on JNK-deficient MEFs demonstrate the requirement of JNK for the release of mitochondrial cytochrome $c$ after UV stimulation (Tournier et al., 2000). JNK-induced apoptosis was found to be largely dependent on the phosphorylation and expression of the Bcl2 protein family (Lei et al., 2002). Sustained, but not transient, JNK activation has also been shown to promote the E3 ubiquitin ligase Itch-mediated degradation of the caspase inhibitor c-FLIP upon TNF $\alpha$ stimulation (Chang et al., 2006). Interestingly, it was proposed that the duration of JNK activation appears to determine the pro- or anti-apoptotic functions. As shown in TNFa-stimulated mouse fibroblasts, the transient phase of JNK activation induces cell survival, whereas prolonged JNK activation mediates apoptotic cascades (Ventura et al., 2006).

\section{p38s}

There are four p38 isoforms in mammalians: $p 38 \alpha, p 38 \beta$, $p 38 \gamma$, and $p 38 \delta$. The $p 38 \alpha$ and $p 38 \beta$ isoforms are widely expressed in most tissues, while $p 38 \gamma$ and $p 38 \delta$ are more strictly expressed in muscle, skin, and kidney cells (Han and Sun, 2007; Hui et al., 2007b). Loss of p38 causes embryonic lethality between embryonic day 10.5 and 16.5 in mice. Impaired vascularization of the placenta was proposed as the cause of death (Adams et al., 2000; Allen et al., 2000; Mudgett et al., 2000; Tamura et al., 2000). Mice with disruption of other p38 isoforms are viable and fertile. Recently, it was reported that mice lacking $p 38 \gamma$ showed defects in insulin secretion in pancreatic $\beta$ cells (Sumara et al., 2009).

p38 $\alpha$ is a serine/threonine kinase which rapidly responds to stresses such as endotoxic lipopolysaccharide (LPS) and heat shock (Han and Sun, 2007; Hui et al., 2007b). p38a phosphorylation is regulated by MKK3 and MKK6, which are activated by upstream kinases, such as MTK1 and ASK1 (Han and Sun, 2007; Hui et al., 2007b). Alternatively, p38a can be activated by a MAPK kinase independent mechanism mediated by TAB1 (Ge et al., 2002). Downstream targets of p38a include protein kinases such as MK2 and PRAK (MK5), and transcription factors such as ATF2 and Mitf (Han and Sun, 2007; Hui et al., 2007b).

Apart from its role in sensing stress signals, recent data also revealed that $p 38 \alpha$ plays a pivotal role in cell cycle transition and cell differentiation (Han and Sun, 2007; Hui et al., 2007b; Wagner and Nebreda, 2009). p38a-deficient MEFs proliferate faster and exhibit delayed senescence compared to control cells, due to the increased G1/S and G2/ $\mathrm{M}$ transitions by upregulation of cyclin D1 and inactivation of p53 activity (Hui et al., 2007a; Sun et al., 2007). Moreover, p38a is shown to be an important regulator of differentiation of various cell types. We recently found p38 $\alpha$ to be required for the terminal differentiation of erythroblasts, probably through enhancing expression of CDK inhibitors p21 and p27 (L.H., unpublished data). During myoblast differentiation, $p 38 \alpha$ regulates the SWI-SNF chromatin-remodeling complexmediated muscle-specific gene expression (Perdiguero et al., 2007). In adult lung tissue, p38a activation is essential to maintain the cell differentiation as well as to restrain proliferation of lung stem and progenitor cells (Ventura et al., 2007).

\section{MAPK SIGNALING IN INFLAMMATION AND CANCER}

The notion that inflammation may lead to tumor development goes back to Virchow's work in the late nineteenth century, who proposed that "lymphoreticulate infiltrate" in chronic inflammatory tissues could be the origin of cancer per se (Balkwill and Mantovani, 2001). A plethora of evidence in epidemiological studies has indicated that chronic inflammation triggered by microbial infection, autoimmune diseases, or other pathologies dramatically raises the risk of tumorigenesis (Mantovani et al., 2008). As an example, hepatitis B and C virus infection is prevalent in hepatocellular carcinoma ( $\mathrm{HCC})$ patients. In China, more than $90 \%$ of $\mathrm{HCC}$ patients are diagnosed with HBV infection. Recent studies using genetically modified mouse models have greatly facilitated the understanding of the causative role of inflammation in cancer cell initiation and progression (Mantovani et al., 2008). Inefficient clearance of infection during chronic inflammation is the major cause of tissue damage and reconstitution. During this process, reactive oxygen species (ROS) accumulate and further lead to DNA damage and mutation. Moreover, cells are continuously proliferating to maintain tissue homeostasis under inflammatory conditions, and this becomes the major driving force for transformation of initial tumor cells (Mantovani et al., 2008). On the other hand, chronic inflammation causes sustained and excessive production of cytokines in advanced tumors. The high levels of cytokines, such as TNF $\alpha$ and IL-6, are key triggers for promoting the malignancy and metastasis of tumor cells (Mantovani et al., 2008). Many studies have demonstrated the critical roles of MAPKs in inflammation and cancer development, and new data show that targeting MAPK pathways appears promising for cancer therapy (Wagner and Nebreda, 2009). 


\section{ERKs in inflammation and cancer}

Active mutations of the Ras-Raf-MEK-ERK module are often found in human cancers and have been demonstrated to facilitate neoplastic transformation. Recent studies revealed that ERK also promotes inflammation-associated cancer development, mainly through regulating the expression of inflammatory cytokines. It was shown that $\mathrm{K}-\mathrm{Ras}^{\mathrm{G} 12 \mathrm{~V}}$-induced pancreatic intraepithelial neoplasia (PanIN) and pancreatic ductal adenocarcinoma (PDA) only develops in the background of mild chronic pancreatitis. Interestingly, phosphorylated ERK is predominantly detected in PanIN and tumor cells of PDA, suggesting a role of ERK in inflammation-related pancreatic cancers (Guerra et al., 2007). Ras activation was demonstrated to elicit local inflammation by enhancing the secretion of CXCL-8 and IL-8, which stimulates neovascularization and tumor growth (Sparmann and Bar-Sagi, 2004). Constitutive activation of the ERK cascade is required for CXCL-8 transcription and contributes to maximal IL-8 gene expression (Sparmann and Bar-Sagi, 2004). Moreover, another report showed that inhibiting MEK activities decreases production of the immunosuppressive soluble factors IL-10, VEGF, or IL-6 in BRAF (V600E) melanoma cells (Sumimoto et al., 2006). The authors concluded that the constitutively active ERK signaling, along with STAT3, is essential for immune evasion by malignant melanoma cells.

\section{JNKs in inflammation and cancer}

Many JNK downstream genes regulate the expression and activation of inflammatory mediators, including TNFa, IL-2, Eselectin and matrix metalloproteinases (Manning and Davis, 2003; Rincon and Davis, 2009). The role of JNK in inflammation has been revealed in a $T$ cell-mediated and TNFa-dependent concanavalin A (ConA) mouse model for acute hepatitis. Induced TNFa in the liver upon ConA treatment results in the activation of JNK, NF-KB and AP-1 (Streetz et al., 2001; Maeda et al., 2003). Both JNK1-null and JNK2-null mouse livers display reduced liver damage after ConA treatment, indicating pivotal roles of JNK in hepatitis (Maeda et al., 2003; Hasselblatt et al., 2007). A recent study using a conditional mouse model showed that mice with JNK $1 / 2$ deletion in the hematopoietic compartment, but not in hepatocytes, exhibit a profound reduction in hepatitis due to reduced TNFa expression (Das et al., 2009). These findings therefore confirm hematopoietic cells to be the site at which JNK plays its role in hepatitis. Besides its role in hepatitis, JNK phosphorylation has been found to be increased at sites of active intestinal inflammation in human patients with inflammatory bowel disease (Mitsuyama et al., 2006). The constitutively activated JNK pathway also greatly promotes detergent-induced colitis in mice (Sancho et al., 2009). Interestingly, deletion of either JNK1 or JNK2 does not prevent the development of colitis in animals (Chromik et al., 2007). However, JNK inactivation using the pan-JNK inhibitor
SP600125 showed a reduction in the production of TNFa, IL-6 and IFN- $\gamma$, and a marked protective effect against experimental intestinal inflammation (Assi et al., 2006), implicating redundant roles of JNKs in colitis.

The oncogenic functions of JNKs in cancer development have been supported by many studies. It was observed that Ras-induced transformation of cultured epithelial cells requires the activation of the JNK pathway (Pruitt et al., 2002). Remarkably, JNK1 has recently been indicated to play a key role in human hepatocellular carcinoma (Chen and Castranova, 2009). Over half of human HCC samples show over-activated JNK1 (Hui et al., 2008). Sustained JNK1 expression in human $\mathrm{HCC}$ contributes to altered histone $\mathrm{H} 3$ methylation, which enhances the expression of genes regulating cell growth (Chang et al., 2009). Using inflammation-associated mouse HCC models, it was confirmed that JNK1 promotes tumor cell proliferation, probably due to the elevated expression of the cell cycle inhibitor p21 and the reduced expression of C-Myc (Hui et al., 2008). In addition, increased JNK activity was found to accelerate the development of chronic colitis-induced colorectal cancer. It was suggested that the control of Tcf4 expression by JNK/cJun probably leads to a positive feedback loop that facilitates colorectal carcinogenesis (Sancho et al., 2009). Furthermore, the JNK pathway is implicated in PI3K-driven human prostate cancer as well. PTEN is frequently found inactivated in human cancer samples. Loss of PTEN increases AKT activity and elevates JNK phosphorylation, which contributes to tumor cell proliferation and angiogenesis (Vivanco et al., 2007). In spite of the oncogenic role of JNK1 in HCC, colon cancer and prostate cancer, JNK1 was reported to function as a tumor suppressor in DMBA/TPA-induced skin tumor and spontaneous colon cancer (She et al., 2002; Tong et al., 2007); however, further analysis of these mouse models might be necessary to clarify the discrepancy.

\section{p38 in inflammation and cancer}

p38 is a pivotal kinase in inflammatory diseases, such as rheumatoid arthritis (RA), psoriasis, and inflammatory bowel disease (Zarubin and Han, 2005; Wagner and Nebreda, 2009). $p 38 \alpha$ and $p 38 \beta$ are predominantly expressed in inflammatory cells. p38 has been shown to be involved in controlling the production of cytokines, such as TNF $\alpha$, IL-1 $\beta$ and IL-6, in the pathogenesis of inflammatory diseases (Wagner and Nebreda, 2009). p38a-null ES cells show diminished production of IL-1-induced IL-6 and fail to activate MAP kinase-activated protein kinase 2 (MK2) in respond to stress stimuli (Allen et al., 2000). The p38a/MK2 pathway is crucial during inflammatory response. Mice lacking MK2 are resistant to the collagen-induced arthritis and show decreased production of inflammatory cytokines (Kotlyarov et al., 1999; Allen et al., 2000). The role of p38a in inflammation has also been proved in mice with tissuespecific deletion of $p 38 \alpha$. The production of LPS-induced 
TNFa, IL-12, and IL-18 is significantly attenuated in macrophages lacking p38a (Kang et al., 2008). Interestingly, a recent study showed that myeloid p38 $\alpha$ activation reduces acute inflammation by inducing anti-inflammatory gene expression in chronic skin inflammation, implicating a double-sided role of p38a in inflammation (Kim et al., 2008).

Two recent reports using conditional $p 38 \alpha$ mice indicate that $p 38 \alpha$ suppresses inflammation-associated liver cancer development (Hui et al., 2007a; Sakurai et al., 2008). In the HCC model used in these studies, carcinogen treatment initiates a wide-range of cell death and tissue repair, similar to the acute phase of hepatitis. High levels of cytokine produced by infiltrated inflammatory cells trigger massive hepatocyte proliferation to maintain tissue homeostasis. p38a was found to suppress proliferation of tumor cells via antagonizing of the JNK-c-Jun pathway (Hui et al., 2007a). Alternatively, it has been proposed that $p 38 \alpha$ can alleviate ROS accumulation and liver damage, thus attenuate proliferation of initial tumor cells in this inflammatory scenario (Sakurai et al., 2008). The tumor suppressive role of $p 38$ has been demonstrated in lung cancer development. A study applying conditional deletion of p38 $\alpha$ in adult mice showed that $\mathrm{K}-\mathrm{Ras}^{\mathrm{G} 12 \mathrm{~V}}$-induced lung tumorigenesis was markedly increased in mutant mice, due to hyperproliferation of lung epithelium and progenitor cells (Ventura et al., 2007). Mice mutated in other components of this pathway revealed similar tumor suppressive functions of p38. Deficiency of the p38a upstream kinase MKK3 or MKK6 in fibroblasts has been found to be associated with increased tumorigenesis (Brancho et al., 2003). Breast tumors triggered by the MMTV-neu oncogene develop at a reduced frequency in mice deficient in Wip1, a p38 $\alpha$ phosphatase (Bulavin et al., 2004). Moreover, a recent study showed a role for PRAK in suppressing skin carcinogenesis by mediating Rasdependent oncogenic senescence upon activation by p38a (Sun et al., 2007).

\section{Crosstalk between MAPK signaling in inflammation and cancer}

The MAPKs are regulated by several common upstream kinases and phosphatases. In addition, various downstream targets, such as AP-1 genes, are shared by MAPKs. Therefore, it is not surprising that these pathways crosstalk with one another in inflammation and cancer development. However, the molecular mechanisms underlying these signaling interactions are largely dependent on cell types and stimulation conditions. A negative crosstalk between the JNK and ERK pathways has been found in TNFa-stimulated cells, in which prolonged JNK phosphorylation uncouples MEK-mediated ERK activation in a c-Jun-dependent manner (Shen et al., 2003). Remarkably, this negative feedback was found in DUSP2-null macrophages and was proposed as an explanation for the reduced inflammatory cytokine expression in these cells (Jeffrey et al., 2006). One of the MAPK crosstalk mechanisms is the suppressive effect of p38a on JNK activation. In a mouse model of chronic skin inflammation, macrophages deficient in p38 $\alpha$ exhibit enhanced and prolonged JNK phosphorylation, which is due to attenuated dual specific phosphatase 1 (DUSP1) expression (Kim et al., 2008). Another work also showed that JNK activities are dramatically increased in p38-deficient livers in LPS-induced hepatitis, although it remains unknown whether increased JNK activities contribute to apoptosis of hepatocytes (Heinrichsdorff et al., 2008). During inflammation-associated liver cancer development, increased activities of the JNK pathway were found to cause the accelerated proliferation rates of liver tumor cells lacking p38a (Hui et al., 2007a). Increased MKK7 phosphorylation was proposed to explain the hyperactivation of JNK in p38a-deficient cancer cells. JNK and p38a could also interact with each other via several other pathways during inflammation and carcinogenesis. TNFa-induced NF$\kappa B$ activation could be another linker between these two MAPKs. As discussed above, TNFa production by inflammatory cells is largely dependent on p38a in chronic inflammation, such as hepatitis. Interestingly, liver cells lacking the NF$\kappa B$ activator IKK $\beta$ show decreased activities of DUSPs, which leads to enhanced JNK1 phosphorylation and increased liver carcinogenesis (Maeda et al., 2005).

\section{PERSPECTIVES}

The roles of MAPKs in tumor initiation and progression have been well documented. A simple model can be proposed as that the MAPK-mediated inflammation plays a role in the initiation of the tumors, while the regulation of cell proliferation, senescence and cell death by MAPKs is essential for the progression of the tumors once they are formed. However, the contribution of MAPKs in cancer initiation and development in human patients, and the molecular mechanisms for MAPKmediated inflammation in tumor growth are still largely unknown. We anticipate that a large number of future studies will aim at previously less well-studied topics such as phosphatases. Application of chemical inhibitors or activators of MAPKs in animal model and clinic trials could be a hotspot in this field.

Intensive studies have been carried out to address the activation of MAPKs; however, inactivation of MAPKs, an important mechanism in controlling the activity of MAPKs, during tumor initiation and progression has been poorly studied. MAPKs can be negatively regulated by a group of DUSPs, all of which harbor an HCxxxxxR protein tyrosine phosphatase (PTP) motif (Patterson et al., 2009). DUSPs inactivate MAPK signaling by removing the phosphate group from the threonine and tyrosine residues. Recent studies in mice deficient in DUSP1, DUSP2 or DUSP10 has defined essential roles of DUSPs in local and systemic inflammation (Lang et al., 2006). For example, DUSP1 has been found to dephosphorylate p38 MAPK in activated macrophages, leading to reduced production of TNFa, IL-6 and IL-10 upon stimulation with TLR-ligands (Lang et al., 2006). In addition, 
DUSP1-deficient mice show high susceptibility to LPSinduced septic shock and collagen-induced arthritis (Lang et al., 2006). Interestingly, DUSP2-deficient macrophages produce attenuated levels of inflammatory mediators, probably due to a negative feedback from the prolonged JNK activation on ERK in these cells (Jeffrey et al., 2006). Until now, studies on DUSP functions in cancer development have mainly involved expression analyses of clinical tumor samples (Keyse, 2008). It remains unknown whether and how DUSPs play roles in tumorigenesis. Nevertheless, since DUSPs negatively regulate MAPK activities in both inflammatory and cancer cells, it is reasonable to speculate that these enzymes would perform important functions in carcinogenesis.

The role of MAPKs in inflammation is primarily due to their ability to control cytokine expression. Since MAPKs participate in cell death processes in a number of pathophysiological conditions, and necrotic cell death can trigger inflammation, the role of MAPKs in inflammation could also be related to cell death. Recent researches have brought autophagy and programmed necrosis (also termed as necropotosis) into the spotlight (Hotchkiss et al., 2009). Autophagy is a process by which cytoplasmic components are sequestered and degraded in a double-membraneorganelle called autophagosome (Virgin and Levine, 2009). An impaired autophagy pathway under stress conditions usually leads to cell death. Autophagy has been associated with various aspects of innate and adaptive immunity, including interferon and cytokine production (Virgin and Levine, 2009). Moreover, autophagy can be subrogated by cancer cells to avoid the adverse micro-environmental stress (Mathew et al., 2007). Recent data has revealed that sustained activation of ERK and p38 plays critical roles in autophagy at the maturation step (Corcelle et al., 2006). However, it remains unclear whether MAPKs regulate autophagy in inflammation-associated tumorigenesis. The latest advances in cell death have revealed that the RIP1 and RIP3 kinases are essential components of a signaling pathway that mediates caspase-independent cell death (necrosis) (Cho et al., 2009; Degterev et al., 2008; He et al., 2009; Zhang et al., 2009). RIPs are well-characterized kinases that transmit TNFa signaling and induce the activation of MAPKs. It is currently unknown whether RIP-mediated activation of MAPKs plays a pro- or anti-death role. Nevertheless, whether MAPKs-mediated cell death contributes to inflammation and subsequent tumor development needs to be addressed in the future.

The roles of MAPKs in inflammatory responses and carcinogenesis have led to the development of a large number of small molecule inhibitors for these kinases. Because the Ras-Raf-MEK-ERK pathway is one of the most frequently activated oncogenic pathway in human cancers, this signaling pathway has been subjected to intensive research to identify novel inhibitors for cancer treatment. Among them, BAY 43-9006 (Raf inhibitor), PD184352,
PD0325901 and ARRY-142886 (MEK1/2 inhibitors) have reached the clinical trial stage (Roberts and Der, 2007). Intriguingly, no inhibitors of ERK1 or ERK2 have been described so far. Given the roles of inflammation in promoting tumor initiation and progression, many pharmaceutical companies have carried out screens for compounds that block cytokine production. As a result, many compounds have been found to inhibit p38s with high selectivity; however, few of them have progressed beyond Phase I clinical trial largely due to toxic side effects. In addition, given the observation that p38a plays a tumor suppressive function in mouse lung and liver cancers, it would be worth exploring whether inhibition of p38a might result in enhanced cancer progression in human patients. On the other hand, it will be interesting to know whether one could activate the p38 pathway, for example, by inhibiting DUSPs, to block tumor growth.

The diverse functions of JNKs in proliferation and cell death are also targets for therapies. A small compound (CC-930) that binds to the JNK ATP-pocket is under clinical trials for lung fibrosis disease by Celgene (Wagner and Nebreda, 2009). The latest published data have shown a shift from identifying an ATP competitive compound to interfering with the interaction between JNK and its scaffold protein JIP1 (Wagner and Nebreda, 2009). To this end, a small peptidebased JNK inhibitor (D-JNKI1) has been tested for degenerative neural disease by Xigen (Wagner and Nebreda, 2009). However, it is not known whether there are JNK inhibitors developed for cancer therapies. Moreover, due to the different functions of JNK1 and JNK2 in cancer development, it is important to develop novel inhibitors specific for JNK1 or JNK2. On the other hand, as the MAPK signaling pathways crosstalk with each other, it should be taken into consideration whether inhibiting a single MAPK gene or a single MAPK pathway would result in unexpected outcomes. One solution for the challenge may lie in the synthetic targeting of different genes. For example, it might be attractive to test whether the combined use of p38 inhibitors that reduce inflammation and JNK inhibitors that decrease cell proliferation would have a more effective outcome for cancer therapy.

\section{ACKNOWLEDGEMENTS}

The work in L.H. laboratory is supported by Shanghai Pujiang Program 09PJ1411200, the Knowledge Innovation Program of Shanghai Institutes for Biological Sciences 2009KIP101, the Knowledge Innovation Program of the Chinese Academy of Sciences KSCX2-YW-R-241, and work in J.H. laboratory is supported by Chinese 973 program 2009CB522200, Chinese NSF grant 30830092 , Chinese-Switzerland collaboration grant 2009DFA32760.

\section{ABBREVIATIONS}

ConA, concanavalin A; DUSP, dual specific phosphatase; ERK, extracellular signal-regulated kinase; HCC, hepatocellular carcinoma; JNK, c-Jun NH2-terminal kinase; LPS, lipopolysaccharide; MAP, 
mitogen-activated protein; MAPK, mitogen activated protein kinase; MK2, MAP kinase-activated protein kinase 2; MKK, MAPK kinase; MAPKK, MAP kinase kinase; MAPKKK, MAP kinase kinase kinase; RA, rheumatoid arthritis; ROS, reactive oxygen species; PanIN, pancreatic intraepithelial neoplasia; PDA, pancreatic ductal adenocarcinoma; PTP, protein tyrosine phosphatase; TEY, Thr-Glu-Tyr

\section{REFERENCES}

Adams, R.H., Porras, A., Alonso, G., Jones, M., Vintersten, K., Panelli, S., Valladares, A., Perez, L., Klein, R., and Nebreda, A.R. (2000). Essential role of p38alpha MAP kinase in placental but not embryonic cardiovascular development. Mol Cell 6, 109-116.

Allen, M., Svensson, L., Roach, M., Hambor, J., McNeish, J., and Gabel, C.A. (2000). Deficiency of the stress kinase p38alpha results in embryonic lethality: characterization of the kinase dependence of stress responses of enzyme-deficient embryonic stem cells. J Exp Med 191, 859-870.

Assi, K., Pillai, R., Gomez-Munoz, A., Owen, D., and Salh, B. (2006). The specific JNK inhibitor SP600125 targets tumour necrosis factor-alpha production and epithelial cell apoptosis in acute murine colitis. Immunology 118, 112-121.

Balkwill, F., and Mantovani, A. (2001). Inflammation and cancer: back to Virchow? Lancet 357, 539-545.

Bost, F., Aouadi, M., Caron, L., Even, P., Belmonte, N., Prot, M., Dani, C., Hofman, P., Pages, G., Pouyssegur, J., et al. (2005). The extracellular signal-regulated kinase isoform ERK1 is specifically required for in vitro and in vivo adipogenesis. Diabetes 54 , 402-411.

Boutros, T., Chevet, E., and Metrakos, P. (2008). Mitogen-activated protein (MAP) kinase/MAP kinase phosphatase regulation: roles in cell growth, death, and cancer. Pharmacol Rev 60, 261-310.

Brancho, D., Tanaka, N., Jaeschke, A., Ventura, J.J., Kelkar, N., Tanaka, Y., Kyuuma, M., Takeshita, T., Flavell, R.A., and Davis, R. J. (2003). Mechanism of p38 MAP kinase activation in vivo. Genes Dev 17, 1969-1978.

Bulavin, D.V., Phillips, C., Nannenga, B., Timofeev, O., Donehower, L. A., Anderson, C.W., Appella, E., and Fornace, A.J., Jr. (2004). Inactivation of the Wip1 phosphatase inhibits mammary tumorigenesis through p38 MAPK-mediated activation of the p16(Ink4a)p19(Arf) pathway. Nat Genet 36, 343-350.

Chambard, J.C., Lefloch, R., Pouyssegur, J., and Lenormand, P. (2007). ERK implication in cell cycle regulation. Biochim Biophys Acta $1773,1299-1310$.

Chang, L., Kamata, H., Solinas, G., Luo, J.L., Maeda, S., Venuprasad, K., Liu, Y.C., and Karin, M. (2006). The E3 ubiquitin ligase itch couples JNK activation to TNFalpha-induced cell death by inducing C-FLIP(L) turnover. Cell 124, 601-613.

Chang, Q., Zhang, Y., Beezhold, K.J., Bhatia, D., Zhao, H., Chen, J., Castranova, V., Shi, X., and Chen, F. (2009). Sustained JNK1 activation is associated with altered histone $\mathrm{H} 3$ methylations in human liver cancer. J Hepatol 50, 323-333.

Chen, F., and Castranova, V. (2009). Beyond apoptosis of JNK1 in liver cancer. Cell Cycle 8, 1145-1147.

Cho, Y.S., Challa, S., Moquin, D., Genga, R., Ray, T.D., Guildford, M., and Chan, F.K. (2009). Phosphorylation-driven assembly of the RIP1-RIP3 complex regulates programmed necrosis and virusinduced inflammation. Cell 137, 1112-1123.
Chromik, A.M., Muller, A.M., Korner, J., Belyaev, O., Holland-Letz, T., Schmitz, F., Herdegen, T., Uhl, W., and Mittelkotter, U. (2007). Genetic deletion of JNK1 and JNK2 aggravates the DSS-induced colitis in mice. J Invest Surg 20, 23-33.

Corcelle, E., Nebout, M., Bekri, S., Gauthier, N., Hofman, P., Poujeol, P., Fenichel, P., and Mograbi, B. (2006). Disruption of autophagy at the maturation step by the carcinogen lindane is associated with the sustained mitogen-activated protein kinase/extracellular signal-regulated kinase activity. Cancer Res 66, 6861-6870.

Das, M., Sabio, G., Jiang, F., Rincon, M., Flavell, R.A., and Davis, R.J. (2009). Induction of hepatitis by JNK-mediated expression of TNFalpha. Cell 136, 249-260.

Degterev, A., Hitomi, J., Germscheid, M., Ch'en, I.L., Korkina, O., Teng, X., Abbott, D., Cuny, G.D., Yuan, C., Wagner, G., et al. (2008). Identification of RIP1 kinase as a specific cellular target of necrostatins. Nat Chem Biol 4, 313-321.

Dong, C., Yang, D.D., Tournier, C., Whitmarsh, A.J., Xu, J., Davis, R. J., and Flavell, R.A. (2000). JNK is required for effector T-cell function but not for T-cell activation. Nature 405, 91-94.

Dumesic, P.A., Scholl, F.A., Barragan, D.I., and Khavari, P.A. (2009). Erk1/2 MAP kinases are required for epidermal G2/M progression. J Cell Biol 185, 409-422.

Eferl, R., and Wagner, E.F. (2003). AP-1: a double-edged sword in tumorigenesis. Nat Rev Cancer 3, 859-868.

Fuchs, S.Y., Dolan, L., Davis, R.J., and Ronai, Z. (1996). Phosphorylation-dependent targeting of c-Jun ubiquitination by Jun N-kinase. Oncogene 13, 1531-1535.

Ge, B., Gram, H., Di Padova, F., Huang, B., New, L., Ulevitch, R.J., Luo, Y., and Han, J. (2002). MAPKK-independent activation of p38alpha mediated by TAB1-dependent autophosphorylation of p38alpha. Science 295, 1291-1294.

Guerra, C., Schuhmacher, A.J., Canamero, M., Grippo, P.J., Verdaguer, L., Perez-Gallego, L., Dubus, P., Sandgren, E.P., and Barbacid, M. (2007). Chronic pancreatitis is essential for induction of pancreatic ductal adenocarcinoma by K-Ras oncogenes in adult mice. Cancer Cell 11, 291-302.

Han, J., and Sun, P. (2007). The pathways to tumor suppression via route p38. Trends Biochem Sci 32, 364-371.

Hasselblatt, P., Rath, M., Komnenovic, V., Zatloukal, K., and Wagner, E.F. (2007). Hepatocyte survival in acute hepatitis is due to c-Jun/ AP-1-dependent expression of inducible nitric oxide synthase. Proc Natl Acad Sci U S A 104, 17105-17110.

Hatano, N., Mori, Y., Oh-hora, M., Kosugi, A., Fujikawa, T., Nakai, N., Niwa, H., Miyazaki, J., Hamaoka, T., and Ogata, M. (2003). Essential role for ERK2 mitogen-activated protein kinase in placental development. Genes Cells 8, 847-856.

He, S., Wang, L., Miao, L., Wang, T., Du, F., Zhao, L., and Wang, X. (2009). Receptor interacting protein kinase-3 determines cellular necrotic response to TNF-alpha. Cell 137, 1100-1111.

Heinrichsdorff, J., Luedde, T., Perdiguero, E., Nebreda, A.R., and Pasparakis, M. (2008). p38 alpha MAPK inhibits JNK activation and collaborates with lkappaB kinase 2 to prevent endotoxininduced liver failure. EMBO Rep 9, 1048-1054.

Hotchkiss, R.S., Strasser, A., McDunn, J.E., and Swanson, P.E. (2009). Cell death. N Engl J Med 361, 1570-1583.

Hui, L., Bakiri, L., Mairhorfer, A., Schweifer, N., Haslinger, C., Kenner, L., Komnenovic, V., Scheuch, H., Beug, H., and Wagner, E.F. (2007a). p38alpha suppresses normal and cancer cell proliferation 
by antagonizing the JNK-c-Jun pathway. Nat Genet 39, 741-749.

Hui, L., Bakiri, L., Stepniak, E., and Wagner, E.F. (2007b). p38alpha: a suppressor of cell proliferation and tumorigenesis. Cell Cycle 6, 2429-2433.

Hui, L., Zatloukal, K., Scheuch, H., Stepniak, E., and Wagner, E.F. (2008). Proliferation of human HCC cells and chemically induced mouse liver cancers requires JNK1-dependent p21 downregulation. J Clin Invest 118, 3943-3953.

Jaeschke, A., Karasarides, M., Ventura, J.J., Ehrhardt, A., Zhang, C., Flavell, R.A., Shokat, K.M., and Davis, R.J. (2006). JNK2 is a positive regulator of the cJun transcription factor. Mol Cell 23, 899-911.

Jeffrey, K.L., Brummer, T., Rolph, M.S., Liu, S.M., Callejas, N.A., Grumont, R.J., Gillieron, C., Mackay, F., Grey, S., Camps, M., et al. (2006). Positive regulation of immune cell function and inflammatory responses by phosphatase PAC-1. Nat Immunol 7, 274-283.

Kang, Y.J., Chen, J., Otsuka, M., Mols, J., Ren, S., Wang, Y., and Han, J. (2008). Macrophage deletion of p38alpha partially impairs lipopolysaccharide-induced cellular activation. J Immunol 180, 5075-5082.

Keyse, S.M. (2008). Dual-specificity MAP kinase phosphatases (MKPs) and cancer. Cancer Metastasis Rev 27, 253-261.

Kim, C., Sano, Y., Todorova, K., Carlson, B.A., Arpa, L., Celada, A., Lawrence, T., Otsu, K., Brissette, J.L., Arthur, J.S., et al. (2008). The kinase p38 alpha serves cell type-specific inflammatory functions in skin injury and coordinates pro- and anti-inflammatory gene expression. Nat Immunol 9, 1019-1027.

Kotlyarov, A., Neininger, A., Schubert, C., Eckert, R., Birchmeier, C., Volk, H.D., and Gaestel, M. (1999). MAPKAP kinase 2 is essential for LPS-induced TNF-alpha biosynthesis. Nat Cell Biol 1, 94-97.

Kuan, C.Y., Yang, D.D., Samanta Roy, D.R., Davis, R.J., Rakic, P., and Flavell, R.A. (1999). The Jnk1 and Jnk2 protein kinases are required for regional specific apoptosis during early brain development. Neuron 22, 667-676.

Lang, R., Hammer, M., and Mages, J. (2006). DUSP meet immunology: dual specificity MAPK phosphatases in control of the inflammatory response. J Immunol 177, 7497-7504.

Lawler, S., Fleming, Y., Goedert, M., and Cohen, P. (1998). Synergistic activation of SAPK1/JNK1 by two MAP kinase kinases in vitro. Curr Biol 8, 1387-1390.

Lei, K., Nimnual, A., Zong, W.X., Kennedy, N.J., Flavell, R.A., Thompson, C.B., Bar-Sagi, D., and Davis, R.J. (2002). The Bax subfamily of $\mathrm{Bcl} 2$-related proteins is essential for apoptotic signal transduction by c-Jun $\mathrm{NH}(2)$-terminal kinase. Mol Cell Biol 22, 4929-4942.

Maeda, S., Chang, L., Li, Z.W., Luo, J.L., Leffert, H., and Karin, M. (2003). IKKbeta is required for prevention of apoptosis mediated by cell-bound but not by circulating TNFalpha. Immunity 19, 725-737.

Maeda, S., Kamata, H., Luo, J.L., Leffert, H., and Karin, M. (2005). IKKbeta couples hepatocyte death to cytokine-driven compensatory proliferation that promotes chemical hepatocarcinogenesis. Cell 121, 977-990.

Manning, A.M., and Davis, R.J. (2003). Targeting JNK for therapeutic benefit: from junk to gold? Nat Rev Drug Discov 2, 554-565.

Mantovani, A., Allavena, P., Sica, A., and Balkwill, F. (2008). Cancerrelated inflammation. Nature 454, 436-444.

Mathew, R., Karantza-Wadsworth, V., and White, E. (2007). Role of autophagy in cancer. Nat Rev Cancer 7, 961-967.
Mazzucchelli, C., Vantaggiato, C., Ciamei, A., Fasano, S., Pakhotin, P., Krezel, W., Welzl, H., Wolfer, D.P., Pages, G., Valverde, O., et al. (2002). Knockout of ERK1 MAP kinase enhances synaptic plasticity in the striatum and facilitates striatal-mediated learning and memory. Neuron 34, 807-820.

Mebratu, Y., and Tesfaigzi, Y. (2009). How ERK1/2 activation controls cell proliferation and cell death: Is subcellular localization the answer? Cell Cycle 8, 1168-1175.

Mitsuyama, K., Suzuki, A., Tomiyasu, N., Tsuruta, O., Kitazaki, S., Takeda, T., Satoh, Y., Bennett, B.L., Toyonaga, A., and Sata, M. (2006). Pro-inflammatory signaling by Jun-N-terminal kinase in inflammatory bowel disease. Int J Mol Med 17, 449-455.

Mudgett, J.S., Ding, J., Guh-Siesel, L., Chartrain, N.A., Yang, L., Gopal, S., and Shen, M.M. (2000). Essential role for p38alpha mitogen-activated protein kinase in placental angiogenesis. Proc Natl Acad Sci U S A 97, 10454-10459.

Pages, G., Guerin, S., Grall, D., Bonino, F., Smith, A., Anjuere, F., Auberger, P., and Pouyssegur, J. (1999). Defective thymocyte maturation in p44 MAP kinase (Erk 1) knockout mice. Science 286, 1374-1377.

Patterson, K.I., Brummer, T., O'Brien, P.M., and Daly, R.J. (2009). Dual-specificity phosphatases: critical regulators with diverse cellular targets. Biochem J 418, 475 489.

Pearson, G., Robinson, F., Beers Gibson, T., Xu, B.E., Karandikar, M., Berman, K., and Cobb, M.H. (2001). Mitogen-activated protein (MAP) kinase pathways: regulation and physiological functions. Endocr Rev 22, 153-183.

Perdiguero, E., Ruiz-Bonilla, V., Gresh, L., Hui, L., Ballestar, E., Sousa-Victor, P., Baeza-Raja, B., Jardi, M., Bosch-Comas, A., Esteller, M., et al. (2007). Genetic analysis of p38 MAP kinases in myogenesis: fundamental role of p38alpha in abrogating myoblast proliferation. EMBO J 26, 1245-1256.

Pruitt, K., Pruitt, W.M., Bilter, G.K., Westwick, J.K., and Der, C.J. (2002). Raf-independent deregulation of p38 and JNK mitogenactivated protein kinases are critical for Ras transformation. J Biol Chem 277, 31808-31817.

Pullikuth, A.K., and Catling, A.D. (2007). Scaffold mediated regulation of MAPK signaling and cytoskeletal dynamics: a perspective. Cell Signal 19, 1621-1632.

Rincon, M., and Davis, R.J. (2009). Regulation of the immune response by stress-activated protein kinases. Immunol Rev 228, 212-224.

Roberts, P.J., and Der, C.J. (2007). Targeting the Raf-MEK-ERK mitogen-activated protein kinase cascade for the treatment of cancer. Oncogene 26, 3291-3310.

Saba-El-Leil, M.K., Vella, F.D., Vernay, B., Voisin, L., Chen, L., Labrecque, N., Ang, S.L., and Meloche, S. (2003). An essential function of the mitogen-activated protein kinase Erk2 in mouse trophoblast development. EMBO Rep 4, 964-968.

Sabapathy, K., Hochedlinger, K., Nam, S.Y., Bauer, A., Karin, M., and Wagner, E.F. (2004). Distinct roles for JNK1 and JNK2 in regulating JNK activity and c-Jun-dependent cell proliferation. Mol Cell 15, 713-725.

Sabapathy, K., Jochum, W., Hochedlinger, K., Chang, L., Karin, M., and Wagner, E.F. (1999). Defective neural tube morphogenesis and altered apoptosis in the absence of both JNK1 and JNK2. Mech Dev 89, 115-124.

Sakurai, T., He, G., Matsuzawa, A., Yu, G.Y., Maeda, S., Hardiman, 
G., and Karin, M. (2008). Hepatocyte necrosis induced by oxidative stress and IL-1 alpha release mediate carcinogen-induced compensatory proliferation and liver tumorigenesis. Cancer Cell 14, 156-165.

Sancho, R., Nateri, A.S., de Vinuesa, A.G., Aguilera, C., Nye, E., Spencer-Dene, B., and Behrens, A. (2009). JNK signalling modulates intestinal homeostasis and tumourigenesis in mice. EMBO J 28, 1843-1854.

She, Q.B., Chen, N., Bode, A.M., Flavell, R.A., and Dong, Z. (2002). Deficiency of C-Jun- $\mathrm{NH}(2)$-terminal kinase-1 in mice enhances skin tumor development by 12-O-tetradecanoylphorbol-13-acetate. Cancer Res 62, 1343-1348.

Shen, Y.H., Godlewski, J., Zhu, J., Sathyanarayana, P., Leaner, V., Birrer, M.J., Rana, A., and Tzivion, G. (2003). Cross-talk between JNK/SAPK and ERK/MAPK pathways: sustained activation of JNK blocks ERK activation by mitogenic factors. J Biol Chem 278, 26715-26721.

Sparmann, A., and Bar-Sagi, D. (2004). Ras-induced interleukin-8 expression plays a critical role in tumor growth and angiogenesis. Cancer Cell 6, 447-458.

Streetz, K., Fregien, B., Plumpe, J., Korber, K., Kubicka, S., Sass, G., Bischoff, S.C., Manns, M.P., Tiegs, G., and Trautwein, C. (2001). Dissection of the intracellular pathways in hepatocytes suggests a role for Jun kinase and IFN regulatory factor-1 in Con A-induced liver failure. J Immunol 167, 514-523.

Sumara, G., Formentini, I., Collins, S., Sumara, I., Windak, R., Bodenmiller, B., Ramracheya, R., Caille, D., Jiang, H., Platt, K.A., et al. (2009). Regulation of PKD by the MAPK p38delta in insulin secretion and glucose homeostasis. Cell 136, 235-248.

Sumimoto, H., Imabayashi, F., Iwata, T., and Kawakami, Y. (2006). The BRAF-MAPK signaling pathway is essential for cancerimmune evasion in human melanoma cells. J Exp Med 203, 1651-1656.

Sun, P., Yoshizuka, N., New, L., Moser, B.A., Li, Y., Liao, R., Xie, C., Chen, J., Deng, Q., Yamout, M., et al. (2007). PRAK is essential for ras-induced senescence and tumor suppression. Cell 128, 295-308.

Tamura, K., Sudo, T., Senftleben, U., Dadak, A.M., Johnson, R., and Karin, M. (2000). Requirement for p38alpha in erythropoietin expression: a role for stress kinases in erythropoiesis. Cell 102, 221-231.

Tong, C., Yin, Z., Song, Z., Dockendorff, A., Huang, C., Mariadason, J., Flavell, R.A., Davis, R.J., Augenlicht, L.H., and Yang, W. (2007).
c-Jun $\mathrm{NH} 2$-terminal kinase 1 plays a critical role in intestinal homeostasis and tumor suppression. Am J Pathol 171, 297-303.

Tournier, C., Dong, C., Turner, T.K., Jones, S.N., Flavell, R.A., and Davis, R.J. (2001). MKK7 is an essential component of the JNK signal transduction pathway activated by proinflammatory cytokines. Genes Dev 15, 1419-1426.

Tournier, C., Hess, P., Yang, D.D., Xu, J., Turner, T.K., Nimnual, A., Bar-Sagi, D., Jones, S.N., Flavell, R.A., and Davis, R.J. (2000). Requirement of JNK for stress-induced activation of the cytochrome c-mediated death pathway. Science 288, 870-874.

Ventura, J.J., Hubner, A., Zhang, C., Flavell, R.A., Shokat, K.M., and Davis, R.J. (2006). Chemical genetic analysis of the time course of signal transduction by JNK. Mol Cell 21, 701-710.

Ventura, J.J., Tenbaum, S., Perdiguero, E., Huth, M., Guerra, C., Barbacid, M., Pasparakis, M., and Nebreda, A.R. (2007). p38alpha MAP kinase is essential in lung stem and progenitor cell proliferation and differentiation. Nat Genet 39, 750-758.

Virgin, H.W., and Levine, B. (2009). Autophagy genes in immunity. Nat Immunol 10, 461-470.

Vivanco, I., Palaskas, N., Tran, C., Finn, S.P., Getz, G., Kennedy, N. J., Jiao, J., Rose, J., Xie, W., Loda, M., et al. (2007). Identification of the JNK signaling pathway as a functional target of the tumor suppressor PTEN. Cancer Cell 11, 555-569.

Wada, T., Joza, N., Cheng, H.Y., Sasaki, T., Kozieradzki, I., Bachmaier, K., Katada, T., Schreiber, M., Wagner, E.F., Nishina, $\mathrm{H}$., et al. (2004). MKK7 couples stress signalling to G2/M cell-cycle progression and cellular senescence. Nat Cell Biol 6, 215-226.

Wagner, E.F., and Nebreda, A.R. (2009). Signal integration by JNK and p38 MAPK pathways in cancer development. Nat Rev Cancer 9, 537-549.

Weston, C.R., and Davis, R.J. (2007). The JNK signal transduction pathway. Curr Opin Cell Biol 19, 142-149.

Yao, Y., Li, W., Wu, J., Germann, U.A., Su, M.S., Kuida, K., and Boucher, D.M. (2003). Extracellular signal-regulated kinase 2 is necessary for mesoderm differentiation. Proc Natl Acad Sci U S A 100, 12759-12764.

Zarubin, T., and Han, J. (2005). Activation and signaling of the p38 MAP kinase pathway. Cell Res 15, 11-18.

Zhang, D.W., Shao, J., Lin, J., Zhang, N., Lu, B.J., Lin, S.C., Dong, M. Q., and Han, J. (2009). RIP3, an energy metabolism regulator that switches TNF-induced cell death from apoptosis to necrosis. Science 325, 332-336. 